\title{
Long-Term Outcomes of Endoscopic Balloon Dilation for Benign Strictures in Patients with Inflammatory Bowel Disease
}

Hye Won Lee ${ }^{1}$, Soo Jung Park ${ }^{1}$, Seong Ran Jeon ${ }^{2}$, Byong Duk Ye ${ }^{3}$, Jae Jun Park ${ }^{4}$, Jae Hee Cheon ${ }^{1}$, Tae II Kim ${ }^{1}$, Won Ho Kim ${ }^{1}$, Korean Association for the Study of the Intestinal Diseases (KASID)

${ }^{1}$ Department of Internal Medicine and Institute of Gastroenterology, Yonsei University College of Medicine, ${ }^{2}$ Institute for Digestive Research, Digestive Disease Center, Soonchunhyang University College of Medicine, ${ }^{3}$ Department of Gastroenterology and Inflammatory Bowel Disease Center, Asan Medical Center, University of Ulsan College of Medicine, and ${ }^{4}$ Department of Internal Medicine, Gangnam Severance Hospital, Yonsei University College of Medicine, Seoul, Korea

Background/Aims: Benign intestinal strictures are common complications in patients with inflammatory bowel disease (IBD). This study aimed to assess the long-term prognosis of endoscopic balloon dilation (EBD) to treat benign strictures in IBD patients. Methods: Patients with IBD who had benign strictures and who underwent EBD in four tertiary referral university hospitals between January 2004 and February 2014 were retrospectively reviewed. Technical success was defined as the ability to pass the scope through the stricture after balloon dilation, and clinical success was defined as improved obstructive symptoms. Results: Forty-two benign strictures were identified in 30 patients ( 15 males and 15 females). Technical success was achieved in 26 patients (86.7\%) at the first EBD attempt and in all 30 patients (100\%) at the second EBD attempt. Clinical success was seen in 28 patients (93.3\%). The median follow-up duration was 134.8 months (range, 10.2 to 252.0 months), and recurrence occurred in eight patients (26.7\%), who required repeat EBD. The median duration to relapse was 1.7 months (range, 0.2 to 6.3 months). During repeat EBD, perforation occurred in two cases $(6.7 \%)$, which were both clipped successfully. Finally, only one patient (3.3\%) underwent surgery for the relief of recurrent obstructive symptoms during the follow-up period. Conclusions: The experience of 10 years shows that EBD is safe and effective for the treatment of benign strictures in IBD patients. Importantly, EBD may allow long-term effective palliation of the symptoms associated with benign intestinal strictures in IBD patients. (Gut Liver 2018;12:530-536)

Key Words: Inflammatory bowel diseases; Crohn disease;
Colitis, ulcerative; Benign stricture; Balloon dilation

\section{INTRODUCTION}

Benign strictures of the gastrointestinal tract are one of the common complications of colorectal disease, particularly in inflammatory bowel disease (IBD) ${ }^{1,2}$ A stricture is defined as the inability to traverse a segment of the intestine with a scope, or a radiographically determined area of luminal narrowing with corresponding obstructive symptoms. Generally, medical treatment including immunomodulator and anti-tumor necrosis factor $\alpha$ (anti-TNF- $\alpha$ ) agents, improve inflammatory strictures. ${ }^{3,4}$ However, fibrotic strictures are often refractory to medical treatment and require surgical resection. Surgery can cause many postoperative complications, including fistulas, leaks, shortbowel syndromes, and frequent stricture recurrence at the anastomosis site. ${ }^{5,6}$ Importantly, the overall stricture recurrence after the first resection in patients with Crohn's disease (CD) is relatively high (50\% to 60\%) during the 15 -year postoperative period. ${ }^{7}$

Strictures of the gastrointestinal tract cause obstructive symptoms such as discomfort, pain, and constipation. Among the many surgical techniques available to treat this condition, bowel-preserving operations, such as strictureplasty, are recommended to minimize short-bowel syndrome. ${ }^{6}$ The recurrence rate of strictures after the first surgical resection is reported to be between 15\% and 45\% within 5 years. ${ }^{8,9}$ To avoid these complications of surgical intervention, endoscopic balloon dilation (EBD) has been attempted in IBD patients with benign strictures, instead of bowel resection in selected cases. ${ }^{10}$ Some studies have

Correspondence to: Soo Jung Park

Department of Internal Medicine and Institute of Gastroenterology, Yonsei University College of Medicine, 50-1 Yonsei-ro, Seodaemun-gu, Seoul 03722, Korea

Tel: +82-2-2228-1963, Fax: +82-2-393-6884, E-mail: sjpark@yuhs.ac

Received on September 1, 2017. Revised on January 8, 2018. Accepted on February 9, 2018. Published online June 22, 2018

pISSN 1976-2283 eISSN 2005-1212 https://doi.org/10.5009/gnl17396

(a) This is an Open Access article distributed under the terms of the Creative Commons Attribution Non-Commercial License (http://creativecommons.org/licenses/by-nc/4.0) which permits unrestricted non-commercial use, distribution, and reproduction in any medium, provided the original work is properly cited. 
reported that EBD is a safe and effective intervention for IBD patients with de novo and anastomotic strictures. ${ }^{11-13}$ However, the patient cohort was composed mainly of CD patients, with few cases of ulcerative colitis (UC). Additionally, data on the long-term outcomes of EBD for benign strictures are still limited. $^{14,15}$

Thus, our aim was to evaluate the long-term prognosis of EBD for benign strictures in patients with IBD. The primary end points were the rates of technical success and clinical success. The secondary end points were the recurrence rate of benign stricture and restenosis prediction factors.

\section{MATERIALS AND METHODS}

\section{Patients}

This study was designed as a multicenter retrospective study of IBD patients with benign strictures treated with EBD. Among the symptomatic IBD patients (including those with CD and UC) from four tertiary university hospitals, those who underwent EBD for benign strictures between January 2004 and February 2014 were considered eligible. Four hospitals included Severance Hospital, Soonchunhyang Hospital, Asan Medical Center and Gangnam Severance Hospital, Seoul, Korea.

All patients had intestinal strictures through which an endoscope could not pass. Among the IBD patients with intestinal strictures, patients with the following conditions were excluded: (1) malignant strictures, (2) no obstructive symptoms, or (3) age less than 18 years. Among the patients who underwent EBD, those who satisfied the following exclusion criteria were further excluded: (1) loss to follow-up after EBD, (2) short follow-up duration ( $<6$ months), or (3) other serious concurrent illness.

Data were collected from four hospitals using a standard protocol. The details included patients' sex, age, disease, concur- rent medication including IBD-related medication for at least 3 months before the endoscopic procedure, number and type of strictures, and complications after EBD. Laboratory tests including erythrocyte sedimentation rate (ESR) and C-reactive protein (CRP) were evaluated at the time of admission for EBD in all patients. This study was approved by an independent Institutional Review Board and conformed to the ethical guidelines of the 1975 Helsinki Declaration (IRB number: 4-2015-0531). The informed consent was waived for this retrospective study.

\section{Procedure}

Before endoscopy, all patients underwent cross-sectional radiologic imaging (abdominopelvic computed tomography [CT] or CT enterography) to confirm the suspected stricture, to exclude intestinal fistulas and angulated strictures, and to measure the length of the strictures. All EBD procedures were carried out by one or two experienced endoscopists in each center, who determined the use of esophagogastroduodenoscopy, colonoscopy, or double-balloon enteroscopy according to the location and type of strictures. Strictures were dilated using a throughthe-scope control radial expansion wire-guided balloon catheter device. The balloon was positioned across the stricture under radiologic guidance. The duration and numbers of balloon inflation varied according to the stricture type.

Technical success was defined as the ability to pass the scope through the stricture after dilation. At the end of the procedure, technical success was confirmed by passing the scope through the dilated stricture site. Clinical success was defined as symptomatic relief after EBD. The relief of obstructive symptoms was also evaluated at least 4 hours after EBD. Repeat dilatations were performed when obstructive symptoms due to restenosis were confirmed with both radiologic imaging and endoscopy.

48 IBD patients with strictures

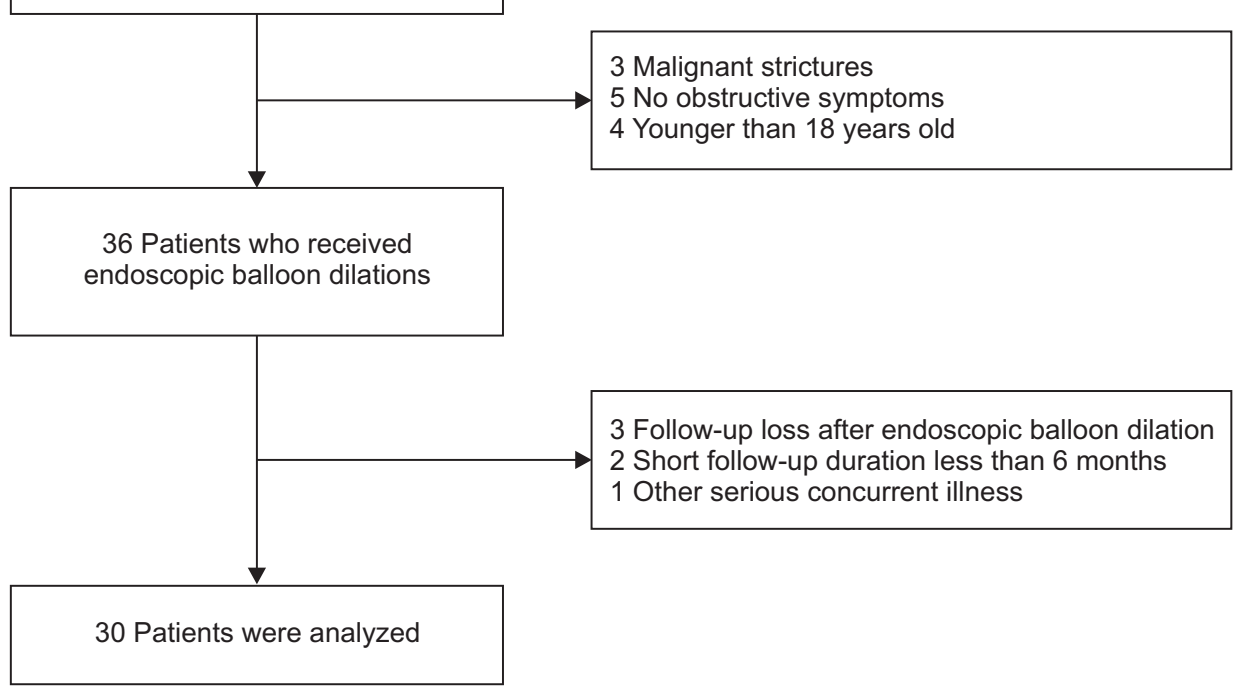

Fig. 1. Patient enrollment algorithm. A total of 48 patients with inflammatory bowel disease (IBD) who underwent endoscopic balloon dilation were enrolled. Patients were excluded based on our exclusion criteria. Finally, a total of 30 patients were selected for the statistical analysis. 


\section{Statistical analysis}

Data are expressed as the mean (standard deviation), median (range), or number (\%), as appropriate. Differences between continuous and categorical variables were examined for statistical significance using a Student t-test (or Mann-Whitney U test, if appropriate) and a chi-square test (or Fisher exact test, if appropriate). Recurrence rates were calculated using the Kaplan-Meier method, and compared with a nonparametric test.

All statistical analyses were performed with the SPSS version 20.0 (IBM Corp., Armonk, NY, USA). A p-value of $<0.05$ was considered statistically significant.

\section{RESULTS}

\section{Baseline characteristics}

As shown in Fig. 1, three patients with malignant strictures, five without symptoms, but definite obstructions that could not pass through the endoscope at all or combined with proximal dilatation in the radiologic studies were noted, four who were $<18$ years old, three with follow-up loss, two with $<6$ month follow-up and a 65-year-old women with severe heart failure who was unable to take care of IBD disease were excluded. Finally, 30 patients were analyzed in this study.

The baseline characteristics of the study population are summarized in Table 1 . Thirty patients (15 males, 15 females) between the ages of 18 and 80 years old with disease duration between 1.2 and 43.8 years (median, 16.5 years) were included in the study. A total of 42 strictures in 30 IBD patients who had received EBD were available for statistical analysis. There were 36 strictures located in the colorectum, five in the small bowel,

Table 1. Baseline Characteristics of the Enrolled Patients $(n=30)$

\begin{tabular}{lc}
\multicolumn{1}{c}{ Variable } & Value \\
\hline Demographic variables & $31.4(18-80)$ \\
Age, yr & $15(50.0)$ \\
Male sex & $25(83.3)$ \\
Crohn's disease & $5(16.7)$ \\
Ulcerative colitis & $16.5(1.2-43.8)$ \\
Duration of IBD, yr & $7(23.3)$ \\
Concomitant medications & $14(46.7)$ \\
Corticosteroids & $10(33.3)$ \\
AZP/6-MP/MTX/tacrolimus & $14(46.7)$ \\
Biologics (infliximab or adalimumab) & $1(3.3)$ \\
5-ASA & $134.8(10.2-252.0)$ \\
None & \\
Duration of follow-up, mo & \\
\hline
\end{tabular}

Data are presented as the median (range) or number (\%). IBD, inflammatory bowel disease; AZP, azathioprine; 6-MP, 6-mercaptopurine; MTX, methotrexate; 5-ASA, 5-aminosalicylic acid. and one in the stomach (Table 2). The most common disease was CD (25 patients, 83.3\%), with an additional five (16.7\%) patients with UC.

\section{Type of strictures}

Of the 42 stricture lesions, 37 (88.1\%) were found in patients with CD and five (11.9\%) were found in patients with UC (Table 2). Stricture length was between 8 and $40 \mathrm{~mm}$ (median, $10 \mathrm{~mm}$ ). The lesions included eight (19.1\%) anastomotic strictures and $34(80.9 \%)$ de novo strictures. Symptoms of obstruction, such as abdominal pain and difficulty in passing stool, were seen in all patients. Fifty-five balloon dilations were performed for the 42 stricture sites: 36 colorectal, three ileal, two jejunal, and one gastric lesion.

\section{EBD outcomes}

Primary technical success was achieved in 26 of the 30 patients (86.7\%) at the first attempt. Fig. 2 showed successful endoscopic procedure before and after EBD. Of the four cases of technically unsuccessful first EBD attempt, technical success after further EBD attempts was achieved within 1 or 2 days. Technical success was achieved in all patients.

Primary clinical success defined as improved in clinical symptoms after attainment of technical success was achieved in 28 patients (93.3\%). These symptoms included abdominal bloating, distention, abdominal pain, nausea, or vomiting were relieved after successful EBD. Two patients with CD did not improve obstructive symptoms after the first procedure. Therefore, the second EBD was performed within 1 week. They finally achieved clinical success after the second EBD. However, one

Table 2. Characteristics of Strictures $(n=42)$

\begin{tabular}{lc}
\hline \multicolumn{1}{c}{ Variable } & Value \\
\hline No. of strictures & $42(1-3)$ \\
Disease with strictures & \\
$\quad$ Crohn's disease & $57(88.1)$ \\
Ulcerative colitis & $55(1-5)$ \\
No. of dilations & \\
Type of stricture & $8(19.1)$ \\
Anastomotic & $34(80.9)$ \\
De novo & \\
Location of stricture & $36(85.7)$ \\
Colorectum & $5(11.9)$ \\
Small bowel & $1(2.4)$ \\
Stomach & $10(8-40)$ \\
Length of stricture, mm & $18(14-25)$ \\
Maximum diameter of the endoscopic & \\
balloon, mm & $30(20-240)$ \\
Duration of balloon inflation, s
\end{tabular}

Data are presented as the median (range) or number (\%). 


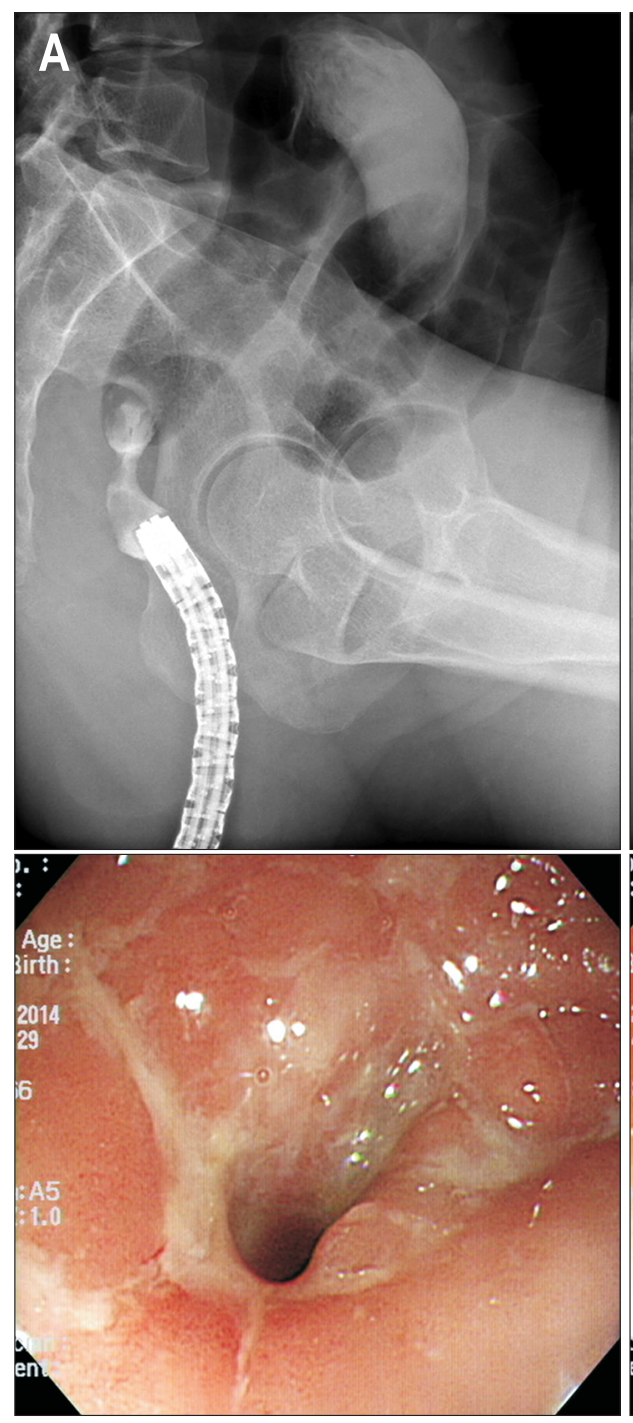

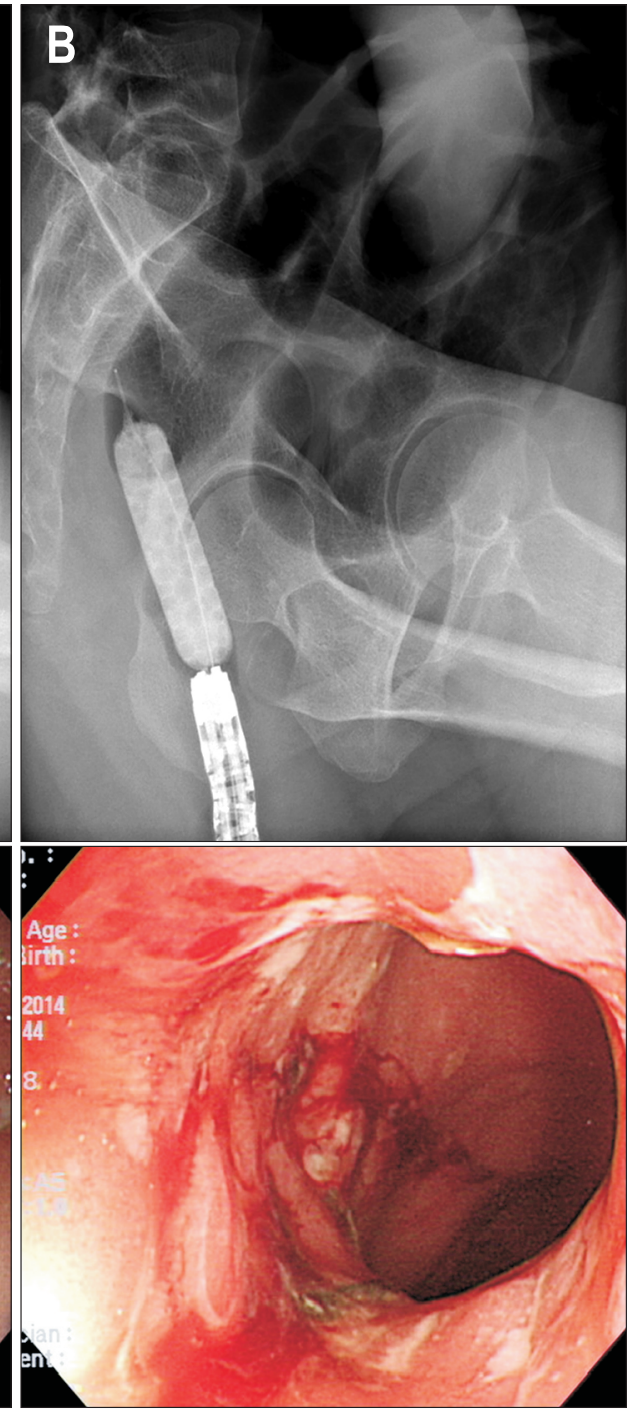

Fig. 2. A case of 39-year-old female Crohn's disease patient with stricture $5 \mathrm{~cm}$ from anal verge (A) before (B) after endoscopic balloon dilation. patient experienced recurrence at 3 months after a first EBD attempt. Despite third EBD attempt, the obstructive symptoms persisted and the patient finally underwent surgery.

Two patients experienced complications after EBD procedure despite clinical or technical success. Two perforations were noted in 55 balloon dilation procedures. They were successfully treated with endoscopic clipping.

\section{Recurrence of benign strictures}

The follow-up duration of patients who underwent EBD was between 10.2 and 252.0 months (median, 134.8 months). Symptomatic strictures recurred in eight patients (26.7\%) and the median time to relapse was 1.7 months (range, 0.2 to 6.3 months). Repeat endoscopic interventions were required in six of the eight patients who had recurrent strictures. Among these, two received a steroid injection at the site of recurrence during the consecutive balloon dilations. One patient had improved symptoms after conservative care, while the other had to have surgical resection owing to recurrence of obstructive symptoms

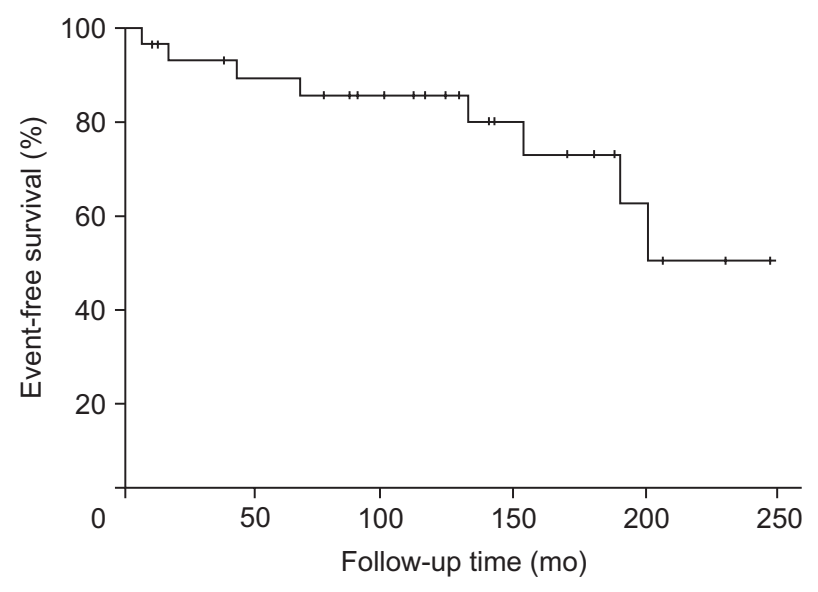

Fig. 3. The cumulative recurrence-free rates of patients without repeat interventions at 12,36 , and 120 months were $96.0 \%, 93.3 \%$, and $86.7 \%$, respectively. 
Table 3. Comparison between Patients with and without Stricture Recurrence

\begin{tabular}{|c|c|c|c|}
\hline Variable & $\begin{array}{c}\text { Patients with recurrence } \\
\qquad(\mathrm{n}=8,26.7 \%)\end{array}$ & $\begin{array}{c}\text { Patients without recurrence } \\
\qquad(\mathrm{n}=22,73.3 \%)\end{array}$ & p-value \\
\hline \multicolumn{4}{|l|}{ Demographic variables } \\
\hline Age, yr & 35.4 & 31.2 & 0.329 \\
\hline Male sex & $4(50.0)$ & $11(50.0)$ & 0.430 \\
\hline Crohn's disease & $6(75.0)$ & 19 (86.4) & 0.004 \\
\hline Ulcerative colitis & $2(25.0)$ & $3(13.6)$ & $<0.001$ \\
\hline Duration of IBD, mo & 15.0 & 19.3 & $<0.001$ \\
\hline De novo stricture & $7(87.5)$ & $15(68.2)$ & 0.029 \\
\hline Location of stricture & & & 0.248 \\
\hline Colorectum & $5(62.5)$ & $19(86.4)$ & \\
\hline Small bowel & $2(25.0)$ & $3(13.6)$ & \\
\hline Stomach & $1(12.5)$ & 0 & \\
\hline Length of stricture, $\mathrm{mm}$ & 16.7 & 9.0 & 0.037 \\
\hline $\mathrm{CRP}, \mathrm{mg} / \mathrm{L}$ & 25.2 & 5.9 & 0.005 \\
\hline $\mathrm{ESR}, \mathrm{mm} / \mathrm{h}$ & 40.3 & 9.8 & $<0.001$ \\
\hline Concomitant medications & & & 0.540 \\
\hline Corticosteroids & $2(25.0)$ & $5(22.7)$ & \\
\hline AZP/6-MP/MTX/tacrolimus & $4(50.0)$ & $10(45.5)$ & \\
\hline Infliximab or adalimumab & $3(37.5)$ & $7(31.8)$ & \\
\hline 5-ASA & $4(50.0)$ & $10(45.5)$ & \\
\hline
\end{tabular}

Data are presented as median or number (\%).

IBD, inflammatory bowel disease; CRP, C-reactive protein; ESR, erythrocyte sedimentation rate; AZP, azathioprine; 6-MP, 6-mercaptopurine; MTX, methotrexate; 5-ASA, 5-aminosalicylic acid.

despite the third EBD attempt at 3 months after the first EBD procedure. The cumulative percentages of patients without repeat endoscopic interventions at 12, 36, and 120 months were 96\%, 93.3\%, and 86.7\%, respectively (Fig. 3).

\section{Comparison between patients with and without recur- rence of symptomatic strictures}

To determine whether there were any predictive factors for recurrence, we analyzed the differences between the recurrence group $(n=8)$ and the non-recurrence group $(n=22)$. We found that the recurrence group had a higher proportion of UC patients (25.0\% vs $13.6 \%, \mathrm{p}<0.001)$, shorter disease duration (15.0 months vs 19.3 months, $\mathrm{p}<0.001$ ), more de novo strictures (87.5\% vs $68.2 \%, p=0.029$ ), longer stricture length (16.7 $\mathrm{mm}$ vs $9.0 \mathrm{~mm}, \mathrm{p}=0.037$ ), higher CRP levels ( $25.2 \mathrm{mg} / \mathrm{L}$ vs $5.9 \mathrm{mg} / \mathrm{L}$, $\mathrm{p}=0.005$ ), and higher ESRs ( $40.3 \mathrm{~mm} / \mathrm{h}$ vs $9.8 \mathrm{~mm} / \mathrm{h}, \mathrm{p}<0.001$ ) than the non-recurrence group (Table 3). There was no significant difference in concomitant medications used between two groups.

\section{DISCUSSION}

Previous studies about the effectiveness of EBD have been conducted, but most follow-up periods have been less than 60 months (range, 17.2-47 months). ${ }^{14-19}$ Although several studies have investigated the efficacy of EBD in treating IBD strictures, no study has specifically evaluated the long-term outcomes of EBD in IBD patients in a multi-center study. Compared to previous studies, this study has the advantage of a long follow-up (approximately 11 years median follow-up) after EBD. We found that in our study cohort, technical success was $86.7 \%$ at the first attempt and 100\% after additional EBD procedures, while clinical success was 93.3\% after the first technical success. The cumulative percentages of patients without repeat endoscopic interventions at 12, 36, and 120 months were 96\%, 93.3\%, and $86.7 \%$, respectively. Together, our study demonstrates that EBD is a safe and effective treatment even after long-term follow-up.

Previous studies have reported that about 33\% of CD patients developed intestinal strictures after 10 years of disease. ${ }^{20,21}$ Compared to $\mathrm{CD}$, colorectal strictures, either benign or malignant, are less common in UC. ${ }^{22}$ Fifty-nine of 1,156 UC patients (5\%) developed 53 benign colorectal strictures between 1,959 and 1,983 . Obstructive symptoms due to intestinal strictures significantly decrease quality of life, and minimally invasive EBD is expected to more effectively treat benign strictures in IBD patients as an alternative to surgical methods. ${ }^{23,24}$

The technical success rate of EBD is reported to be between $70 \%$ and $90 \%$ (follow-up duration ranges from 17.2 months to 
29.4 months). ${ }^{16-18}$ One meta-analysis demonstrated a technical success rate of 90\% and a surgery-free rate ranging from 53\% to $92 \%$, which is consistent with our results. ${ }^{25}$ More than one dilation is usually required for strictures in IBD patients because of recurrence, commonly caused by persistent inflammation. In our study, patients with recurrence showed that $\mathrm{CD}$ was more frequent, de novo stricture was greater, length of stricture was longer, and the degree of inflammation was higher compared to those without. Consistent with this, it is generally believed that IBD strictures may be more resistant to endoscopic dilation than non-IBD strictures. As a secondary measure, inflammatory strictures in IBD patients can be treated with steroids or TNF- $\alpha$ antagonists. ${ }^{26,27}$

In several studies, endoscopic balloons with an external diameter of 18 to $25 \mathrm{~mm}$ were used to dilate strictures of the lower gastrointestinal tract; we used endoscopic balloons with a diameter of 14 to $25 \mathrm{~mm}$. The usual complication was perforation, particularly when 25-mm balloons were used. Most cases of perforation have been reported to occur at the ileocolonic anastomosis site. ${ }^{28,29}$ In our study, perforations occurred in a 60-year-old UC woman with UC and a 31-year-old woman with $\mathrm{CD}$ and were successfully managed by endoscopic clipping. Both patients had left colonic lesions for which expansion was attempted for 30 seconds using a balloon diameter of $18 \mathrm{~mm}$.

The predictive factors for the long-term outcomes of EBD vary among studies. A recent study showed that primary and anastomotic CD strictures had similar rates of surgical and additional EBD. ${ }^{27}$ In another study, anastomotic strictures were associated with improved surgery-free survival. ${ }^{14}$ It is controversial whether intralesional corticosteroid or anti-TNF antibody injections into strictures after dilation improve clinical outcomes. Some studies reported that intralesional corticosteroid injection after EBD prevents re-strictures. ${ }^{29-31}$ However, other studies showed that intralesional corticosteroid injection did not reduce the need for re-dilation or surgery. ${ }^{27,32}$ Monoclonal anti-TNF antibodies are widely used for IBD that is refractory to conventional therapy, or for prophylaxis after surgery. ${ }^{33}$ The effect of anti-TNF therapy on the progression of strictures has not been fully investigated. ${ }^{34,35}$ However, one study suggested that injecting infliximab during EBD significantly reduced the risk of surgery. ${ }^{36}$

This study has some limitations. First, the number of patients was still small despite being a multicenter study. We did not find any independent predictive factors for stricture recurrence, which may be due to the small number of patients and strictures. Second, we did not perform further analysis regarding the outcome of local injection therapies, such as steroids or antiTNF antibodies. Third, due to the retrospective nature of the study, there were no standardized protocols for the insufflation technique, minimal number of dilations, optimal characteristics of strictures, and appropriate diameter for avoiding major complications. Lastly, few strictures occurred in the small bowel, likely because the data analyzed were obtained before doubleballoon endoscopy had been broadly implemented. Moreover, no patient underwent balloon dilation for stenosis due to intestinal tuberculosis or intestinal Behçet's disease owing to the rarity of these diseases. However, although our study has limitations, it also has the strength of having a relatively long-term follow-up duration (median, 11.2 years).

In conclusion, the long-term (median, 134.8 months) technical and clinical success rates of EBD are remarkable compared with those of surgery. EBD may is effective and safe for treating benign IBD strictures. Additionally, EBD may provide long-term effective palliation of symptoms and prevent the need for surgical resection in IBD patients with symptomatic strictures.

\section{CONFLICTS OF INTEREST}

No potential conflict of interest relevant to this article was reported.

\section{ACKNOWLEDGEMENTS}

The authors are grateful to Dong-Su Jang (Medical Illustrator, Medical Research Support Section, Yonsei University College of Medicine, Seoul, Korea) for the help with this journal.

\section{REFERENCES}

1. Ferlitsch A, Reinisch W, Püspök A, et al. Safety and efficacy of endoscopic balloon dilation for treatment of Crohn's disease strictures. Endoscopy 2006;38:483-487.

2. Park CH, Yoon JY, Park SJ, et al. Clinical efficacy of endoscopic treatment for benign colorectal stricture: balloon dilatation versus stenting. Gut Liver 2015;9:73-79.

3. Nielsen $\mathrm{OH}$. New strategies for treatment of inflammatory bowel disease. Front Med (Lausanne) 2014;1:3.

4. Sokol H, Seksik P, Cosnes J. Complications and surgery in the inflammatory bowel diseases biological era. Curr Opin Gastroenterol 2014;30:378-384.

5. Alexander-Williams J, Haynes IG. Conservative operations for Crohn's disease of the small bowel. World J Surg 1985;9:945-951.

6. Lee EC, Papaioannou N. Minimal surgery for chronic obstruction in patients with extensive or universal Crohn's disease. Ann R Coll Surg Engl 1982;64:229-233.

7. Wolters FL, Russel MG, Stockbrügger RW. Systematic review: has disease outcome in Crohn's disease changed during the last four decades? Aliment Pharmacol Ther 2004;20:483-496.

8. Legnani PE, Kornbluth A. Therapeutic options in the management of strictures in Crohn's disease. Gastrointest Endosc Clin N Am 2002;12:589-603.

9. Blomberg B, Rolny P, Järnerot G. Endoscopic treatment of anastomotic strictures in Crohn's disease. Endoscopy 1991;23:195-198.

10. Paine E, Shen B. Endoscopic therapy in inflammatory bowel dis- 
eases (with videos). Gastrointest Endosc 2013;78:819-835.

11. Hassan C, Zullo A, De Francesco V, et al. Systematic review: endoscopic dilatation in Crohn's disease. Aliment Pharmacol Ther 2007;26:1457-1464.

12. Stienecker K, Gleichmann D, Neumayer U, Glaser HJ, Tonus C. Long-term results of endoscopic balloon dilatation of lower gastrointestinal tract strictures in Crohn's disease: a prospective study. World J Gastroenterol 2009;15:2623-2627.

13. Vrabie R, Irwin GL, Friedel D. Endoscopic management of inflammatory bowel disease strictures. World J Gastrointest Endosc 2012;4:500-505.

14. Endo K, Takahashi S, Shiga H, Kakuta Y, Kinouchi Y, Shimosegawa T. Short and long-term outcomes of endoscopic balloon dilatation for Crohn's disease strictures. World J Gastroenterol. 2013;19:86-91.

15. de'Angelis N, Carra MC, Borrelli 0, et al. Short- and long-term efficacy of endoscopic balloon dilation in Crohn's disease strictures. World J Gastroenterol 2013;19:2660-2667.

16. Thomas-Gibson S, Brooker JC, Hayward CM, Shah SG, Williams CB, Saunders BP. Colonoscopic balloon dilation of Crohn's strictures: a review of long-term outcomes. Eur J Gastroenterol Hepatol 2003;15:485-488.

17. Breysem Y, Janssens JF, Coremans G, Vantrappen G, Hendrickx G, Rutgeerts P. Endoscopic balloon dilation of colonic and ileocolonic Crohn's strictures: long-term results. Gastrointest Endosc 1992;38:142-147.

18. Scimeca D, Mocciaro F, Cottone M, et al. Efficacy and safety of endoscopic balloon dilation of symptomatic intestinal Crohn's disease strictures. Dig Liver Dis 2011;43:121-125.

19. Morar PS, Faiz O, Warusavitarne J, et al. Systematic review with meta-analysis: endoscopic balloon dilatation for Crohn's disease strictures. Aliment Pharmacol Ther 2015;42:1137-1148.

20. Louis E, Collard A, Oger AF, Degroote E, El Yafi FA, Belaiche J. Behaviour of Crohn's disease according to the Vienna classification: changing pattern over the course of the disease. Gut 2001;49:777-782.

21. Oberhuber G, Stangl PC, Vogelsang H, Schober E, Herbst F, Gasche C. Significant association of strictures and internal fistula formation in Crohn's disease. Virchows Arch 2000;437:293-297.

22. Gumaste V, Sachar DB, Greenstein AJ. Benign and malignant colorectal strictures in ulcerative colitis. Gut 1992;33:938-941.

23. Wolters FL, Russel MG, Sijbrandij J, et al. Disease outcome of inflammatory bowel disease patients: general outline of a Europewide population-based 10-year clinical follow-up study. Scand J Gastroenterol Suppl 2006;(243):46-54.
24. Krauss E, Agaimy A, Gottfried A, et al. Long term follow up of through-the-scope balloon dilation as compared to strictureplasty and bowel resection of intestinal strictures in crohn's disease. Int J Clin Exp Pathol 2014;7:7419-7431.

25. Wibmer AG, Kroesen AJ, Gröne J, Buhr HJ, Ritz JP. Comparison of strictureplasty and endoscopic balloon dilatation for stricturing Crohn's disease: review of the literature. Int J Colorectal Dis 2010;25:1149-1157.

26. De Cruz P, Kamm MA, Hamilton AL, et al. Crohn's disease management after intestinal resection: a randomised trial. Lancet 2015;385:1406-1417.

27. Atreja A, Aggarwal A, Dwivedi S, et al. Safety and efficacy of endoscopic dilation for primary and anastomotic Crohn's disease strictures. J Crohns Colitis 2014;8:392-400.

28. Couckuyt H, Gevers AM, Coremans G, Hiele M, Rutgeerts P. Efficacy and safety of hydrostatic balloon dilatation of ileocolonic Crohn's strictures: a prospective longterm analysis. Gut 1995;36:577-580.

29. Ramboer C, Verhamme M, Dhondt E, Huys S, Van Eygen K, Vermeire L. Endoscopic treatment of stenosis in recurrent Crohn's disease with balloon dilation combined with local corticosteroid injection. Gastrointest Endosc 1995;42:252-255.

30. Brooker JC, Beckett CG, Saunders BP, Benson MJ. Long-acting steroid injection after endoscopic dilation of anastomotic Crohn's strictures may improve the outcome: a retrospective case series. Endoscopy 2003;35:333-337.

31. Di Nardo G, Oliva S, Passariello M, et al. Intralesional steroid injection after endoscopic balloon dilation in pediatric Crohn's disease with stricture: a prospective, randomized, double-blind, controlled trial. Gastrointest Endosc 2010;72:1201-1208.

32. East JE, Brooker JC, Rutter MD, Saunders BP. A pilot study of intrastricture steroid versus placebo injection after balloon dilatation of Crohn's strictures. Clin Gastroenterol Hepatol 2007;5:10651069.

33. Condino G, Calabrese E, Zorzi F, et al. Anti-TNF-alpha treatments and obstructive symptoms in Crohn's disease: a prospective study. Dig Liver Dis 2013;45:258-262.

34. Thienpont C, Van Assche G. Endoscopic and medical management of fibrostenotic Crohn's disease. Dig Dis 2014;32 Suppl 1:35-38.

35. Chang CW, Wong JM, Tung CC, Shih IL, Wang HY, Wei SC. Intestinal stricture in Crohn's disease. Intest Res 2015;13:19-26.

36. Ono Y, Hirai F, Matsui T, et al. Value of concomitant endoscopic balloon dilation for intestinal stricture during long-term infliximab therapy in patients with Crohn's disease. Dig Endosc 2012;24:432438. 